\title{
Nuclear and Spindle Dynamics in Budding Yeast $\mathbf{v}$
}

\author{
Sidney L. Shaw, Paul Maddox, Robert V. Skibbens, Elaine Yeh, \\ E. D. Salmon, and Kerry Bloom
}

Department of Biology, University of North Carolina at Chapel Hill, Chapel Hill, North Carolina

27599-3280

Monitoring Editors: Jennifer Lippincott-Schwartz and W. James Nelson

\section{INTRODUCTION}

The wealth of information from genetic and more recently genomic studies of the budding yeast has been staggering. However, the small size of the organism has limited traditional cytological approaches. Thus there is a disparity in our detailed understanding of the genetic control of mitosis, for instance, relative to the morphology of mitosis. The application of timelapse high-resolution digital-enhanced differential interference contrast (DE-DIC) and multimode fluoresence microscopy to studying yeast cell division has revealed a very dynamic process that reflects the interplay among microtubule dynamics, microtubulebased motor proteins, and positional determinants in the mother and bud (Salmon et al., 1998a,b; Shaw et al., 1997a,b).

\section{VIDEO SEQUENCES}

\section{Video Sequence 1: DE-DIC of wild-type mitosis}

This video sequence demonstrates the dynamics of nuclear movement, the rates and biphasic nature of spindle pole elongation (anaphase B), and morphological changes in the nucleus during anaphase and the timing of cell cycle progression (Figure 1).

A pair of arrows on each frame indicates the spindle orientation. A and B show spindle assembly. A short spindle, spanning the nucleus is evident. $C$ shows preanaphase spindle bisects nucleus. D shows insertion of the nucleus through the neck. E shows spindle elongation into the bud. F shows bilobed nuclei in mother and bud. G shows completion of spindle elongation. In $\mathrm{H}$, the nucleus moves from the distal site in the mother to the cell center. I shows cytokinesis followed by cell separation.

The average cell cycle is $125 \pm 9 \mathrm{~min}$. Cells spend $\sim 54 \mathrm{~min}$ in $\mathrm{G}_{1}$ and $\mathrm{S}$ phase (I-A). Shortly after $\mathrm{S}$ phase a bipolar spindle is formed and persists at the $2-\mu \mathrm{m}$ stage for $\sim 16 \mathrm{~min}$ (B and C). Anaphase onset (marked by spindle pole separation in D) through maximal

$\nabla$ Online version of this essay contains video information for Figures 1-7. Online version available at www.molbiolcell.org.

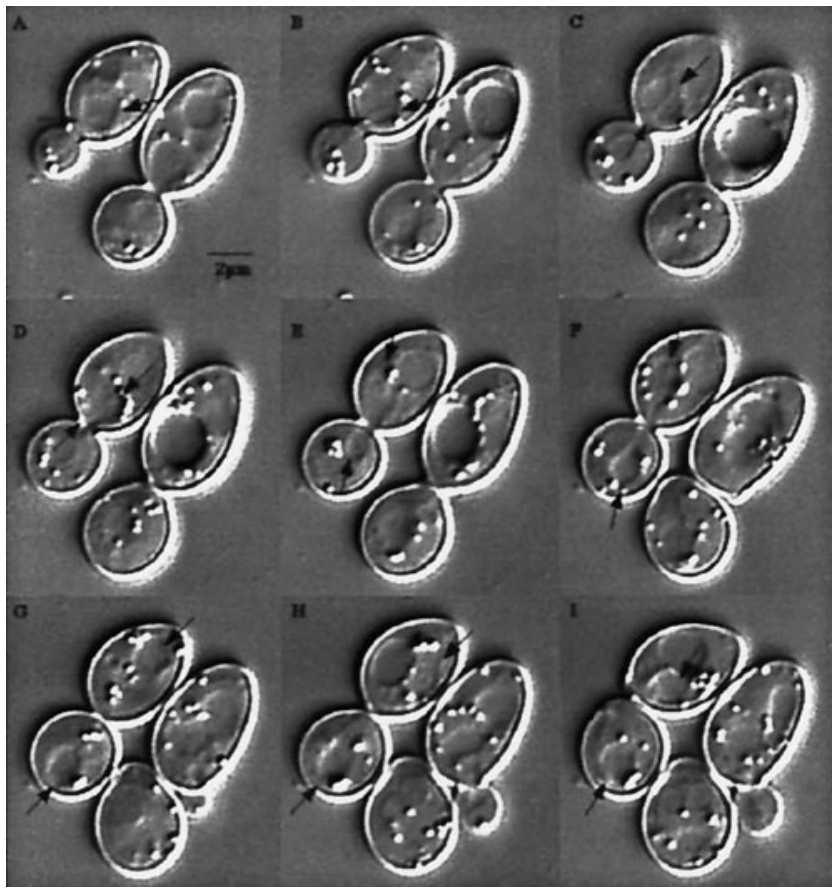

Figure 1. Video sequence 1: DE-DIC of wild-type mitosis.

spindle elongation $(G)$ takes $30 \mathrm{~min}$. Anaphase B is biphasic, characterized by a fast phase $(\sim 1 \mu \mathrm{m} / \mathrm{min})$, followed by slow phase at about one-third the rate (also see Kahana et al., 1995). The time from late anaphase/telophase to cell separation (G-I, marked by mother and bud snapping apart in $\mathrm{H}$ and I) is $\sim 25 \mathrm{~min}$ (data summarized from Yeh et al., 1995).

\section{Video Sequence 2: A Schematic Representation of Microtubule Dynamics and Nuclear Movement Throughout the Cell Cycle in Budding Yeast.}

Tagging expressed proteins with green fluorescent protein (gfp) is a highly specific and sensitive technique for studying the intracellular dynamics of proteins and organelles. We have fused gfp to the carboxyl terminus of the microtubule-based motor 


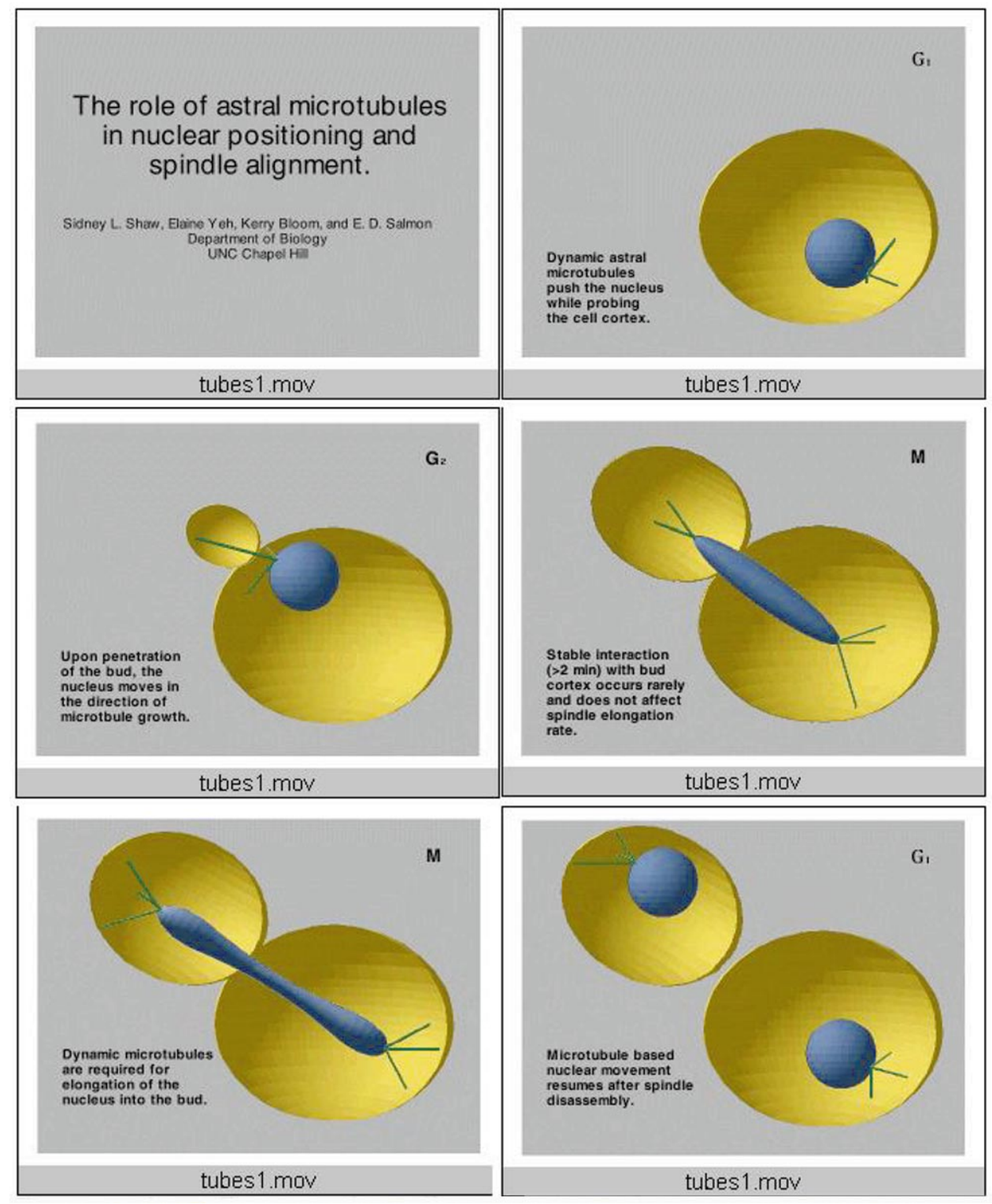

Figure 2. Video sequence 2: microtubule dynamics and nuclear movement throughout the cell cycle in budding yeast.

protein cytoplasmic dynein. Dynein-gfp exclusively labels the astral microtubules in budding yeast. Timelapse fluorescence microscopy provided the first views of astral microtubule dynamics in live yeast cells and revealed several new aspects of microtubule function (Figure 2) (Shaw et al., 1997a,b; see also Carminati and Stearns, 1997; Straight et al., 1997, for microtubules labeled with Tub1-GFP).

\section{Video Sequence 3: Microtubule Growth in $G_{1}$ Is Coupled to Nuclear Movement}

Dynamic astral microtubules from a single spindle pole body (SPB) push against the cell cortex and pro- pel the nucleus in the opposite direction (Figure 3). High-resolution DIC overlain with fluorescence (right) and fluorescence only (left) images of unbudded $\mathrm{G}_{1}$ cells are shown. Haploid cells lack an endogenous copy of dynein and contain dynein-GFP. The focus of fluorescence represents the SPB, which could be seen at the edge of the nucleus in DIC (right). A series of 5 -min time points at 1-min intervals is displayed from top to bottom. Nuclear movement is from left to right over the time course. The cytoplasmic microtubules were organized into a cone-shaped array facing away from the nucleus. Note the SPB at the leading edge of the nucleus and the cytoplasmic microtubules grow- 


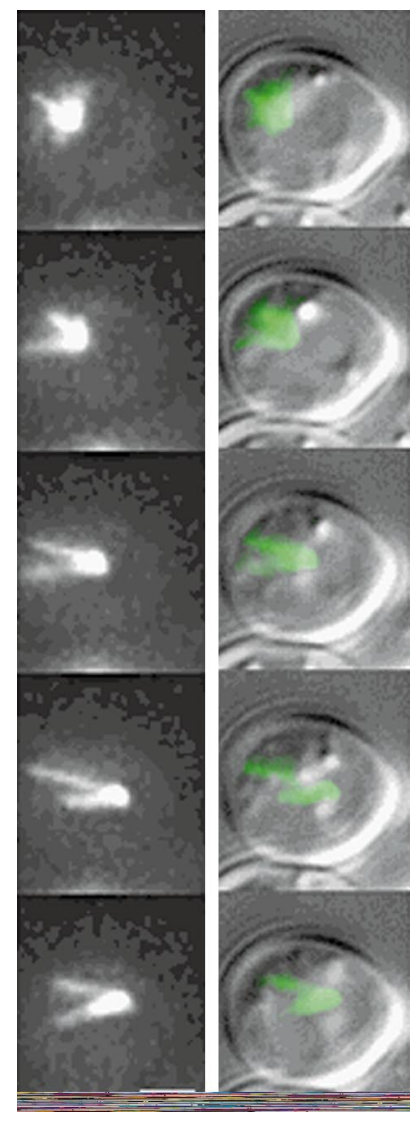

Figure 3. Video sequence 3: microtubule growth in $\mathrm{G}_{1}$ is coupled to nuclear movement. Bar, $2 \mu \mathrm{m}$.

ing opposite to the direction of movement. As an individual microtubule (or microtubule arrays) extended to the left, the nucleus was propelled rightward.

\section{Video Sequence 4: Microtubule Penetration in the Bud Precedes Nuclear Movement to the Bud}

The ability of astral microtubules to find the bud and orient the spindle along the mother-bud axis is critical in a cell that chooses the site of cell division before making a mitotic spindle. Astral microtubules are reported to be oriented toward the site of bud growth before bud emergence. However, as shown in the time-lapse DE-DIC images, the nucleus is very dynamic and moves with a high degree of freedom in unbudded cells in a direction away from microtubule growth. Imaging of astral microtubules with dyneingfp reveals that only after cytoplasmic astral microtubules penetrate the bud does the nucleus migrate to the bud neck (Figure 4). Unbudded haploid cells containing dynein-GFP were captured in the process of bud emergence. A series of DIC (right) and fluorescence (left) images over the time course indicated in

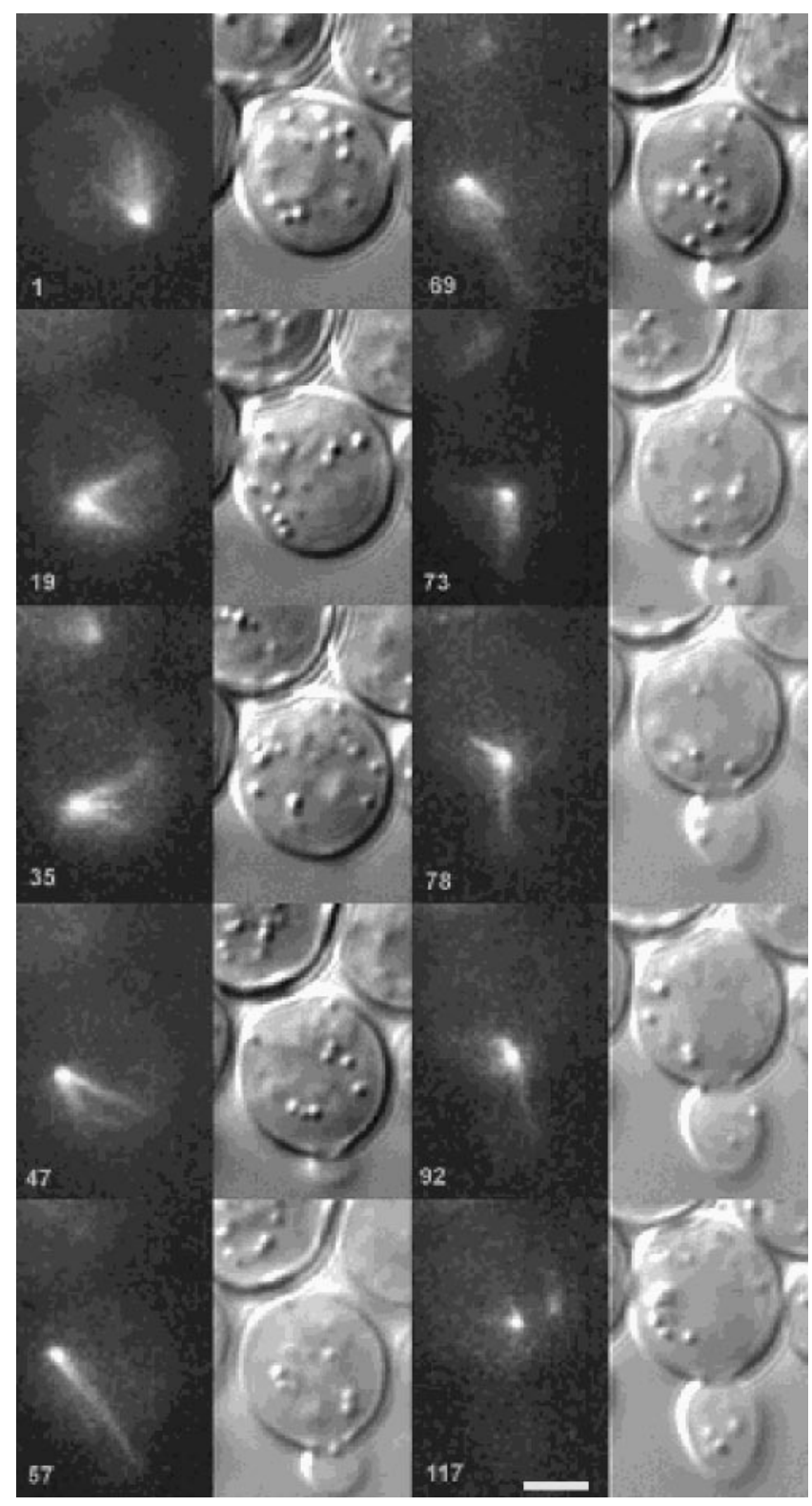

Figure 4. Video sequence 4: microtubule penetration in the bud precedes nuclear movement to the bud. Bar, $2 \mu \mathrm{m}$.

the bottom left (in minutes) is shown from top to bottom, in consecutive series. In the top left quadrant, the unbudded cell has a single SPB (embedded in the nuclear envelope), that is positioned at $\sim 5$ o'clock. In the next sequence, the SPB has migrated to $\sim 7$ o'clock. Note that the astral microtubules trail the SPB and form a conical array. The position of bud emergence is 6 o'clock (see $47 \mathrm{~min}$ ). The SPB migrates around the cell periphery until the $9-10$ o'clock position (47 $\mathrm{min}$ ), well past the emerging bud. At this time, a long microtubule $(7 \mu \mathrm{m})$ can be seen extended toward and into the bud. The nucleus migrates in the direction of 


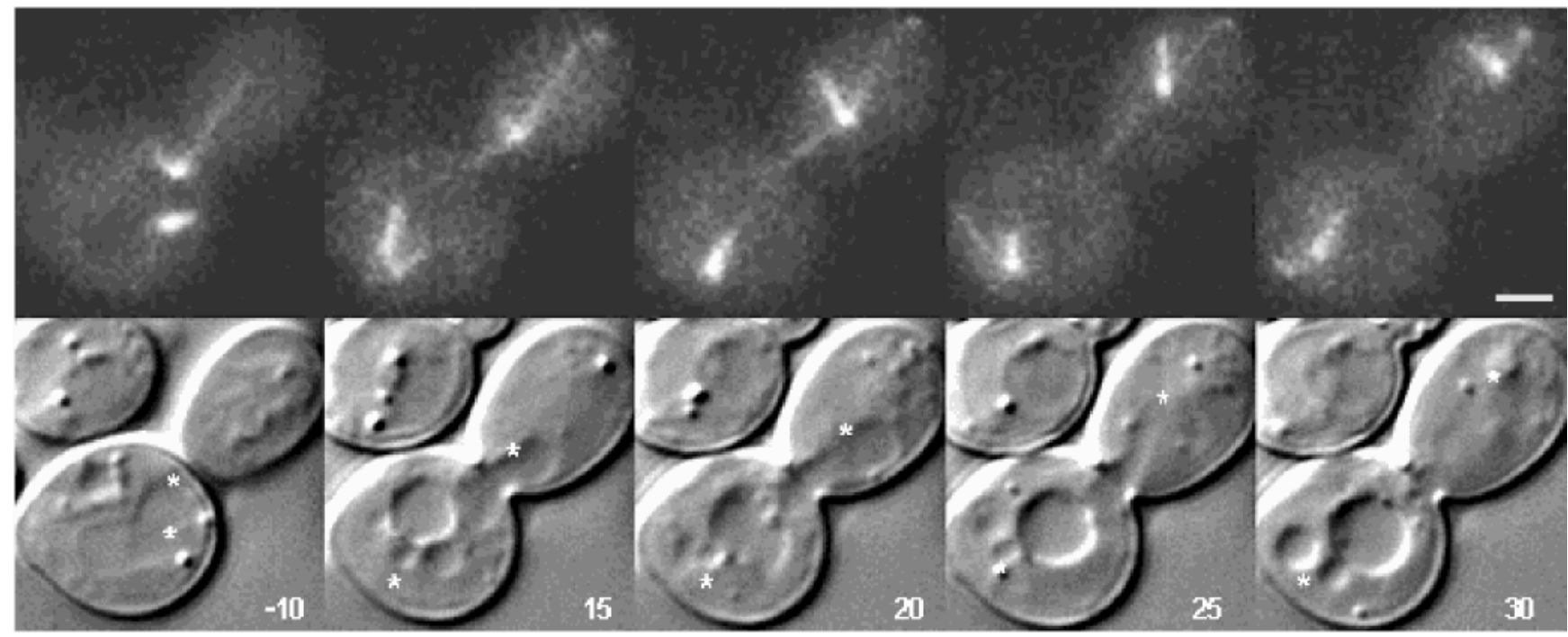

Figure 5. Video sequences 5 and 6: differential timing of the dynein-gfp accumulation of spindle poles. Bar, $2 \mu \mathrm{m}$.

the bud after microtubule penetration of the bud (57-69 $\mathrm{min}$ ). The nucleus continues toward the bud neck, in the direction of the microtubule over the next 23 min (69-92 min). Fluorescently labeled SPBs were apparent at $117 \mathrm{~min}$.

\section{Video Sequence 5: Differential Timing of Dynein-gfp Accumulation on Spindle Poles}

Each cell is born with a single SPB. After SPB duplication, the astral microtubules originate from a bridge structure between the two spindle poles (Byers and Goetsch, 1975). Once the SPBs separated during spindle formation, cytoplasmic dynein remained associated with one pole, typically the pole destined for the bud (see movie). There was a temporal delay $(\sim 10$ min) after visible separation of the spindle poles before the second SPB accumulated cytoplasmic dynein.

\section{Video Sequences 5 and 6: Spindle Elongation in Anaphase}

Figure 5 shows DIC (bottom) and fluorescence (top) images demonstrating the sequence of anaphase SPB separation. Two SPBs (stored in DIC) of equal intensity can be seen in the top left $10 \mathrm{~min}$ before anaphase onset. The SPB closest to the neck is destined for the bud. The nucleus can be seen in DIC where the positions of the spindle poles have been denoted by an asterisk. By $10 \mathrm{~min}$, the fast portion of anaphase is complete, and by 15 min after anaphase onset, a microtubule from one SPB extends into the bud to the tip of the budded cell. Note that the nucleus spans the neck at this time point (15 min after anaphase onset) and that the spindle pole does not lead the nucleus. The microtubule in the bud shortens with spindle elongation (5-min intervals, 15-25 min). The cytoplasmic microtubules associated with the SPB destined for the mother cell remain short and distant from the cortex as the SPB moves toward the base of the mother cell. The cytoplasmic microtubules associated with SPB that are not in contact with the cortex remain dynamic. Note that upon spindle disassembly the nuclei in both the

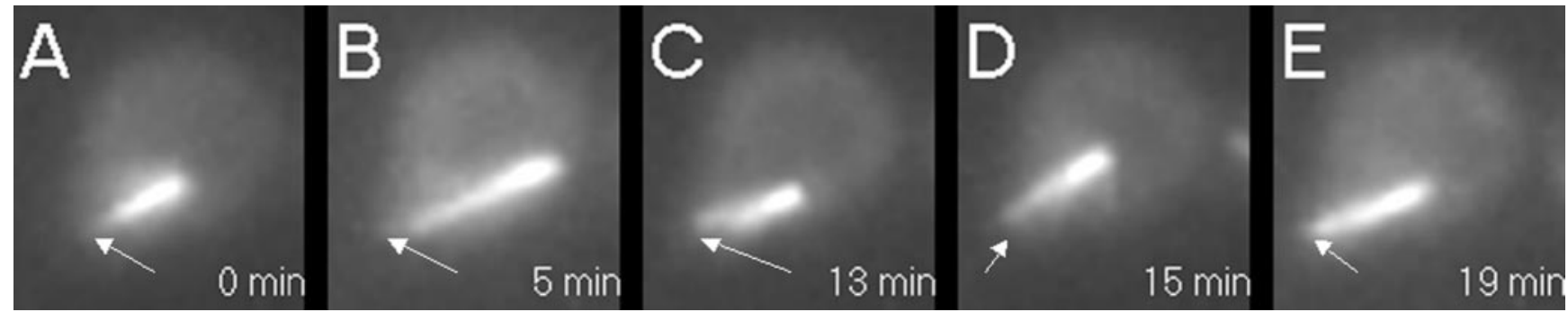

Figure 6. Video sequence 6: spindle elongation in anaphase. 


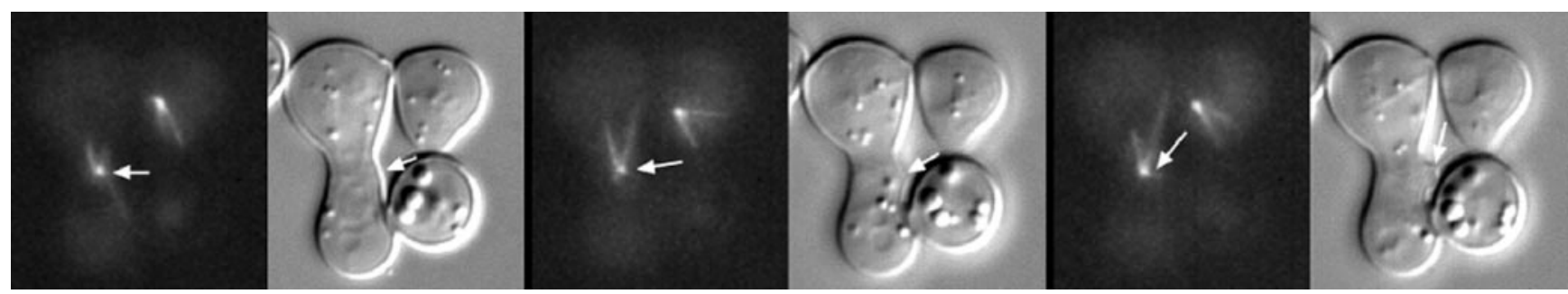

Figure 7. Video sequence 7: behavior of astral microtubules in the process of mating.

mother and bud cell returned to the $G_{1}$ movement typified by growing microtubules that propel the nucleus opposite to the direction of microtubule growth.

\section{Video Sequences 6 and 7: Behavior of Astral Microtubules in the Process of Mating}

Microtubules appear to be stabilized preferentially at the tip of the mating projection (Figure 6). The dynamic attachment of astral microtubules leads to oscillations of the SPB and nucleus toward and away from the mating projection. The rate of oscillation mirrors the dynamics of cytoplasmic microtubules. Thus astral microtubules exhibit transient but persistent interactions with a restricted site in the cell cortex that is the site of cellular deformation preceding cell fusion.

After cell fusion, the nuclei congress toward one another (keryogamy), bringing the spindle poles into close proximity (Mating2.tif; Figure 7). The spindle poles fuse (indicated by the arrow in the fluorescent image), and astral microtubules can be seen in the top cell. The site of spindle pole body fusion determines the site of bud emergence in the first zygotic division (arrow in DIC image indicates the site of bud growth). As the poles separate upon the initiation of anaphase there is a linear kinetic progression ( $1 \mu \mathrm{m} / \mathrm{min}$ ) through telophase.

\section{REFERENCES}

Byers, B., and Goetsch, L. (1975). Behavior of spindles and spindle plaques in the cell cycle and conjugation of Saccharomyces cerevisiae. J. of Bacteriol. 124, 511-523.

Carminati, J.L., and Stearns, T. (1997). Microtubules orient the mitotic spindle in yeast through dynein-dependent interactions with the cell cortex. J. Cell Biol. 138, 629-641.

Kahana, J.A., Schnapp, B.J., and Silver, P.A. (1995). Kinetics of spindle pole body separation in budding yeast. Proc. Natl. Acad. Sci. USA 92, 9707-9711.

Salmon, E.D., Shaw, S.L., Waters, J., Waterman-Storer, C., Maddox, P.S., Yeh, E., and Bloom, K. (1998a). Multimode fluorescence microscopy. Methods Cell Biol. 56, 186-216.

Salmon, E.D., Yeh, E., Shaw, S.L., Skibbens, R., and Bloom, K.S (1998b). High resolution VE- and DE-DIC light microscopy of cell division in budding yeast. Methods Enzymol. 298, 317-331.

Shaw, S.L., Yeh, E., Bloom, K., and Salmon, E.D. (1997a). Imaging GFP-fusion proteins in Saccharomyces cerevisiae. Curr. Biol. 7, 701704.

Shaw, S.L., Yeh, E., Maddox, P., Salmon, E.D., and Bloom, K. (1997b). Astral microtubule dynamics in yeast: A microtubule-based searching mechanism for spindle orientation and nuclear migration in the bud. J. Cell Biol. 139, 985-994.

Straight, A.F., Marshall, W.F., Sedat, J.W., and Murray, A.W. (1997). Mitosis in living budding yeast: anaphase A but no metaphase plate. Science 227, 574-578.

Yeh, E., Skibbens, R., Cheng, J., Salmon, E.D., and Bloom, K. (1995). Spindle dynamics and cell cycle regulation of cytoplasmic dynein in the yeast, S. cerevisiae. J. Cell Biol. 130, 687-700. 